\title{
Viscoelastic Behavior of Scarcely Crosslinked Poly(dimethyl siloxane) Gel
}

\author{
Hideaki TAKAHASHI,,${ }^{* *}$ Yoshitaka ISHIMURO, ${ }^{*}$ and Hiroshi WATANABE ${ }^{* *}$ \\ * Toray Research Center Inc., Sonoyama, Otsu, Shiga 520-8567, Japan \\ ** Institute for Chemical Research, Kyoto University, Uji, Kyoto 611-0011, Japan \\ (Received : December 12, 2005)
}

\begin{abstract}
Linear viscoelastic behavior was investigated for a poly(dimethyl siloxane) (PDMS) gel formed through a bulk double-liquid crosslinking reaction of two types of vinyl-terminated PDMS prepolymers of the molecular weights $M_{\text {pre }} \cong$ $35 \times 10^{3}$. Time-temperature superposition worked, and master curves at $20^{\circ} \mathrm{C}$ were constructed for the storage and loss moduli, $G^{\prime}$ and $G^{\prime \prime}$, in a wide range of angular frequency $\omega\left(=10^{3}-10^{-5} \mathrm{~s}^{-1}\right)$ by combining the data obtained from dynamic oscillatory tests and creep tests. With decreasing $\omega$ to $10^{-1} \mathrm{~s}^{-1}, G^{\prime \prime}$ decreased in proportion to $\omega^{0.6}$ and $G^{\prime}$ rapidly decreased to its equilibrium plateau at the modulus $G_{\mathrm{e}}=2800 \mathrm{~Pa}$. On a further decrease of $\omega$ well in the plateau regime of $G^{\prime}, G^{\prime \prime}$ decreased in proportion to $\omega^{0.3}$. Thus, the gel exhibited the fast and slow relaxation processes characterized with these types of power-law behavior of $G^{\prime \prime}$. The molecular weight between the crosslinks evaluated from the $G_{\mathrm{e}}$ data (as well as the equilibrium swelling ratio in toluene), $M_{\mathrm{c}} \cong 340 \times 10^{3}$, was about ten times larger than $M_{\text {pre }}$. The crosslinking reaction was made in the bulk state but still gave such a scarce gel network (with $M_{\mathrm{c}} \cong 10 M_{\text {pre }}$ ) possibly because a large amount of sol chains and dangling chains had diluted the trapped entanglements during the reaction. From the analysis of the $G^{\prime}$ and $G^{\prime \prime}$ data on the basis of the above $M_{\mathrm{c}}$ value and the intrinsic Rouse relaxation time, the fast relaxation process was assigned as the Rouse-like constraint release (CR) process of individual gel strands. The polydispersity of the strands was found to be essential for the power-law behavior $\left(G^{\prime \prime} \propto \omega^{n}\right.$ with $\left.\mathrm{n} \cong 0.6\right)$ to be observed in the plateau regime of $G^{\prime}$. The slow relaxation process was related to fluctuation of the crosslinking points, which is equivalent to cooperative Rouse-CR motion of many gel strands connected at these points.
\end{abstract}

Key Words: Poly(dimethyl siloxane) / Viscoelastic solids / Scarce gel / Constraint release

\section{INTRODUCTION}

So-called "silicone gels" and "silicone rubbers", composed of polymer networks containing siloxane backbone, have been widely utilized in the industrial fields of electronics and automobile because of their excellent electrical property (high resistance) and chemical stability at high temperatures. The use of the "silicone gels" in the field of cosmetics is also being planned, because these gels are soft and serve as an excellent barrier against water.

These "silicone gels" and "silicone rubbers" are mostly prepared through a crosslinking reaction of poly(dimethyl siloxane) (PDMS) prepolymers. The network structure in these materials can be tuned fairly easily through a control of the molecular weight and a chemical modification of the prepolymers. Thus, the PDMS gels/rubbers serve as important model network systems free from a strain-induced crystallization that often disturbs studies of natural rubbers. For example, the critical gelation behavior ${ }^{1)}$ and the fractal structure on this gelation ${ }^{2,3)}$ have been investigated for PDMS gels, and the swelling dynamics ${ }^{4}$ and the distance between the crosslinks $^{5)}$ have been examined with dynamic/static scattering methods. Studies have been also made for an effect of molecular weight distribution of the PDMS prepolymer on the rubber elasticity after crosslinking ${ }^{6}$, an effect of dangling chains on the elasticity and swelling behavior ${ }^{7}$, a relationship of the nonlinear viscoelastic property and the network structure $^{8,9)}$, and the scattering behavior under large deformation. ${ }^{10)}$ Recently, Urayama et al. ${ }^{11-15)}$ made a series of studies for well characterized PDMS rubbers/gels to demonstrate interesting features of these rubbers/gels: In particular, they analyzed the energy density function of carefully prepared PDMS rubbers to find a significant contribution from the non-Mooney-Rivlin terms under large deformation. ${ }^{13)}$ Furthermore, they synthesized soft PDMS rubbers through a crosslinking reaction in fairly dilute solutions and showed that these rubbers in the dry state are enormously extensible because of the super-coiled structure of 
the network strands therin. ${ }^{11)}$ They also synthesized PDMS rubbers with a temperature-insensitive loss tangent by controlling the heterogeneity in the network structure. ${ }^{15)}$

Rubber elasticity has been also subjected to theoretical studies for more than five decades. For example, Flory ${ }^{16)}$ and James and Guth ${ }^{17)}$ indicated that the fluctuation of the crosslinking points reduces the equilibrium modulus. Later, Langley $^{18)}$ and Dossin and Graessley ${ }^{19)}$ demonstrated that the modulus is enhanced by the trapped entanglement (permanent knot) between the network strands, and Murakami et al. ${ }^{20)}$ proposed a chemo-rheological method for determining the molecular weight between crosslinks. Recently, Urayama et al. ${ }^{14)}$ made an extensive test of molecular models for rubber elasticity and demonstrated that a version of the slip-link model (Edwards-Vilgis model ${ }^{21)}$ ) describes most successfully the experimental data in a wide range of strain for various modes of deformation (e.g., uniaxial and biaxial elongation).

With this background, this study focuses on the linear viscoelastic behavior of a PDMS gel network prepared with a double-liquid crosslinking reaction of two types of vinylterminated prepolymers in bulk. Analyses of the viscoelastic data suggested that this reaction, utilizing neither solvents nor multi-functional crosslinkers and thus differing from the reactions in the previous studies, enabled us to prepare a very soft gel network having a surprisingly small mechanical loss. In addition, this gel exhibited weak but very slow relaxation modes that may be related to the gel network dynamics. Details of these results are presented/discussed in this paper.

\section{EXPERIMENTAL}

\subsection{Material}

Commercially available two vinyl-terminated PDMS prepolymers (SE1886; Toray-Dow Corning Silicone Co., Ltd) were used. The number- and weight-average molecular weights of one prepolymer (neat PDMS having vinyl groups at the ends) were $M_{\mathrm{n}}=13.2 \times 10^{3}$ and $M_{\mathrm{w}}=34.7 \times 10^{3}$, respectively. The other prepolymer (chemically modified prepolymer), with a small fraction $(0.7 \mathrm{~mol} \%)$ of its methyl group being substituted by hydrogen, had $M_{\mathrm{n}}=11.5 \times 10^{3}$ and $M_{\mathrm{w}}=35.1 \times 10^{3}$.

The two prepolymers were mixed at $1 / 1 \mathrm{wt} / \mathrm{wt}$ ratio and cast in a metal mold of an inner diameter of $\sim 20 \mathrm{~mm}$ and height of $\sim 2 \mathrm{~mm}$. This mixture was allowed to react at $120{ }^{\circ} \mathrm{C}$ for $3 \mathrm{~h}$ to prepare a PDMS gel. Some details of this double-liquid crosslinking reaction (catalyzed with a trace amount of $\mathrm{Pt}$ complex included in the neat PDMS prepolymer) are shown in Scheme 1: The vinyl group at a prepolymer end reacts with the monomethyl siloxane group in the chemically modified prepolymer $^{22)}$ as well as with the end vinyl group of the other prepolymer. The former route of the reaction, possibly occurring more frequently than the latter ${ }^{22}$, gives a dangling prepolymer grafted onto a middle of the other prepolymer if the reaction efficiency is low, which was the case for the gel examined in this paper.

Since a large fraction of a sol component was left after the reaction, this paper refers to our material as a gel, rather than a rubber, although it contained no low- $M$ solvent. (The sol component effectively behaved as a solvent for the PDMS network, as discussed later.)

\subsection{Measurements}

For the PDMS gel specimen prepared as above as well as for the prepolymers, dynamic oscillatory measurements were made with a laboratory rheometer (MR-500 Soliquid-meter; Reoroji Co., Ltd) to determine the storage and loss moduli, $G^{\prime}(\omega)$ and $G^{\prime \prime}(\omega)$, in a range of angular frequency $\omega=0.063$ $63 \mathrm{~s}^{-1}$ at various temperatures $T$ between $-40{ }^{\circ} \mathrm{C}$ and $100{ }^{\circ} \mathrm{C}$. A parallel-plate fixture of a diameter $=20 \mathrm{~mm}$ was used. For the gel specimen, the measurement was made under slight compression (with a normal stress $<0.15 \mathrm{~Pa}$ ). The linearity of the $G^{\prime}$ and $G^{\prime \prime}$ data was confirmed by varying the oscillatory strain amplitude.
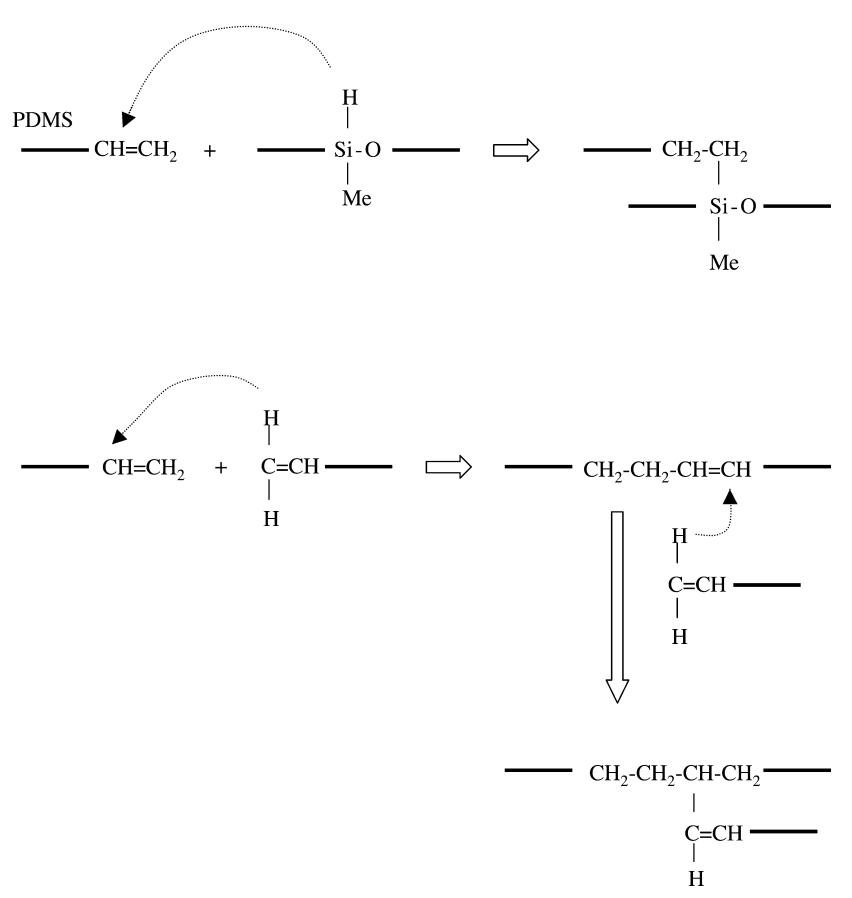

Scheme 1. Double-liquid crosslinking reaction of two types of vinyl terminated PDMS prepolymers in bulk. The upper route of the reaction gives a dangling prepolymer grafted onto a middle of the other prepolymer if the reaction efficiency is low. 
For the PDMS gel specimen, a creep test was also conducted with a laboratory stress rheometer (RS600; Haake Co., Ltd) to determine the creep compliance $J(t)$ at $20{ }^{\circ} \mathrm{C}$. A parallel-plate fixture of a diameter $=20 \mathrm{~mm}$ was used, and the measurement was conducted at a shear stress of $50 \mathrm{~Pa}$ that was well in the linear viscoelastic regime for the gel. The $J(t)$ data were converted to $G^{\prime}(\omega)$ and $G^{\prime \prime}(\omega)$ data on the basis of the linear viscoelastic relationship ( $c f$. Appendix A).

For convenience of construction of master curves of the $G^{\prime}$ and $G^{\prime \prime}$ data of the PDMS gel, changes of its density with $T$ were determined at various $T$ (between $-40{ }^{\circ} \mathrm{C}$ and $100{ }^{\circ} \mathrm{C}$ ) from a measurement of the linear thermal expansion coefficient made with a thermal equipment (CN8098D1; Rigaku Denki Co., Ltd). The measurement was conducted at a heating rate of $2 \mathrm{Kmin}^{-1}$ under a constant load of $0.5 \mathrm{~g}$ wt.

The as-prepared PDMS gel was also subjected to a swelling experiment. After the viscoelastic tests, the gel was soaked in a good solvent, toluene, at room temperature for $48 \mathrm{~h}$, with the solvent being exchanged once in every $12 \mathrm{~h}$. The sol component was thoroughly extracted in the toluene phase after this soaking procedure. The mass of the remaining gel was determined after full removal of toluene through vacuum evacuation. The mass of the sol component extracted in the supernatant toluene phase was determined from a concentrationcalibration of this phase made with a GPC equipment (Model510; Waters Co., Ltd) connected with a differential refractive index detector (RI-8020; Tosoh Co., Ltd).

In addition, for the extracted sol component, the molecular weight and its distribution were determined with GPC and low-angle light scattering (LALS) measurements, the latter utilizing a LALS photometer (KMX-6; Chromatrix Co., Ltd). These measurements were also conducted for respective PDMS prepolymers.

\section{RESULTS AND DISCUSSION}

\subsection{Relaxation Behavior of PDMS Prepolymers}

Figure 1 shows the $G^{\prime}$ and $G^{\prime \prime}$ data at $20^{\circ} \mathrm{C}$ obtained for the neat PDMS prepolymer. For the other, chemically modified prepolymer (with a partial substitution of the methyl group by hydrogen), the data were close to those shown here because the two prepolymers had nearly the same molecular weight and molecular weight distribution (MWD). Clearly, the prepolymers exhibit the terminal flow behavior characterized with the power-law dependencies, $G^{\prime} \propto \omega^{2}$ and $G^{\prime \prime} \propto \omega$.

The terminal relaxation time $\tau_{\mathrm{w}}$ of these prepolymers was determined from those $G^{\prime}$ and $G^{\prime \prime}$ data as

$$
\tau_{\mathrm{w}} \equiv J_{\mathrm{e}} \eta_{0}=\left(\frac{G^{\prime}}{\omega G^{\prime \prime}}\right)_{\omega \rightarrow 0}
$$

Here, $J_{\mathrm{e}}$ and $\eta_{0}$ are the steady state compliance and zero-shear viscosity, respectively. Figures $2 \mathrm{a}$ and $2 \mathrm{~b}$, respectively, compare the $\tau_{\mathrm{w}}$ and $\eta_{0}$ data of the prepolymers with those of high- $M$, narrow-MWD PDMS $\left(M_{\mathrm{w}}>10^{5}\right.$ and $\left.M_{\mathrm{w}} / M_{\mathrm{n}}<1.2\right)$, the latter being evaluated from the $G^{\prime}$ and $G^{\prime \prime}$ data reported in literature. ${ }^{23)}$ Since our prepolymers have a broad MWD $\left(M_{\mathrm{w}} /\right.$ $M_{\mathrm{n}}=2.6$ and 3.1), their $\tau_{\mathrm{w}}$ should be considerably longer than that of narrow-MWD PDMS having the same $M_{\mathrm{w}}{ }^{24,25)}$ Thus, in Fig. 2a, the $\tau_{\mathrm{w}}$ data of the prepolymers have been corrected for their MWD with a method explained in Appendix B. The $\tau_{\mathrm{w}}$ values after this correction were:

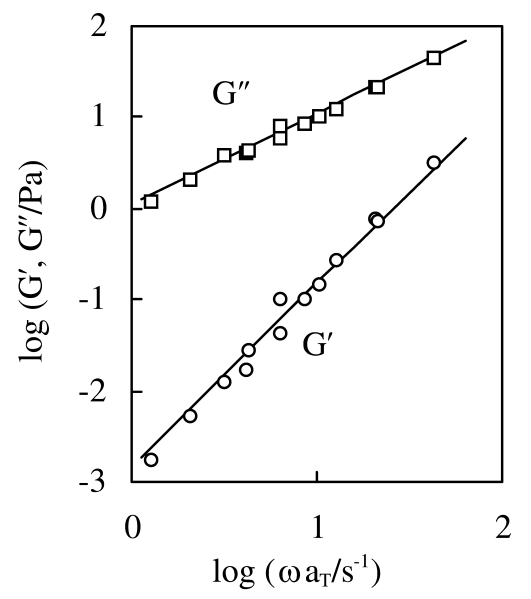

Fig. 1. Dependence of storage modulus $\left(G^{\prime}\right)$ and loss modulus $\left(G^{\prime \prime}\right)$ on angular frequency $(\omega)$ measured for neat PDMS prepolymer (without chemical modification) at $20^{\circ} \mathrm{C}$.
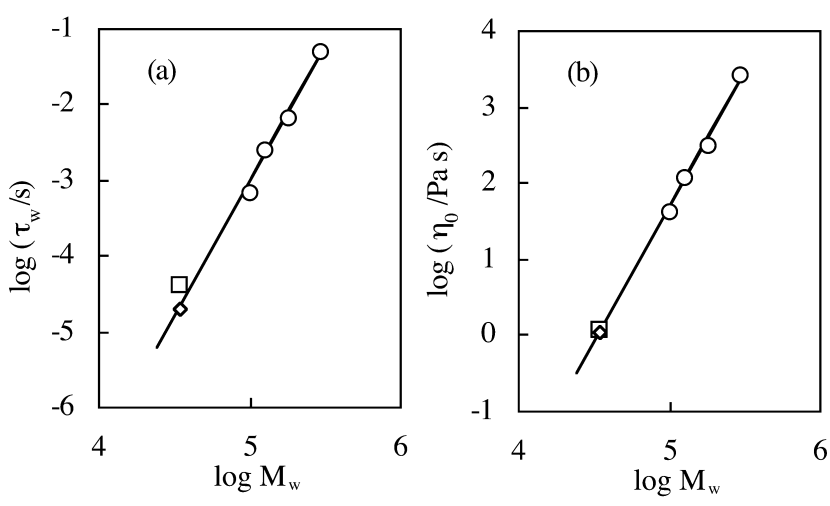

Fig. 2. Molecular weight dependence of (a) terminal relaxation time $\tau_{\mathrm{w}}$ and (b) zero-shear viscosity $\eta_{0}$ of well entangled linear PDMS chains at $20{ }^{\circ} \mathrm{C}$ reported in literature (circles). ${ }^{23)}$ Squares and diamonds indicate the $\tau_{\mathrm{w}}$ and $\eta_{0}$ data of the PDMS prepolymers without and with chemical modification, respectively. For these prepolymers, the $\tau_{\mathrm{w}}$ data shown in the left panel have been corrected for the molecular weight distribution, while no correction has been made for the $\eta_{0}$ data (right panel). 
$\tau_{\mathrm{w}}=4.2 \times 10^{-5} \mathrm{~s} \quad$ for neat PDMS prepolymer

$\tau_{\mathrm{w}}=2.1 \times 10^{-5} \mathrm{~s} \quad$ for chemically modified PDMS prepolymer

In contrast, the $\eta_{0}$ data are insensitive to MWD and essentially determined by $M_{\mathrm{w}}{ }^{24,25)}$ Thus, no correction of MWD has been made for the $\eta_{0}$ data of prepolymers shown in Fig. $2 \mathrm{~b}$.

For PDMS homopolymers, the characteristic molecular weights for the onset of the entanglement effect on $\eta_{0}$ and $J_{\mathrm{e}}$ are reported to be $M_{\eta}=24.4 \times 10^{3}$ and $M_{\mathrm{J}}=61.0 \times 10^{3}$, respectively. ${ }^{24)}$ Thus, the high- $M$ PDMS chains examined in Fig. 2 (circles) are in the well entangled state $\left(M_{\mathrm{w}}>M_{\mathrm{J}}\right)$ and exhibit a typical power-law relationships ${ }^{24,25)}$ shown with the solid lines:

$$
\begin{aligned}
& \tau_{\mathrm{w}}=1.0 \times 10^{-21} M_{\mathrm{w}}^{3.6} \text { (in s) at } 20^{\circ} \mathrm{C} \\
& \eta_{0}=5.0 \times 10^{-17} M_{\mathrm{w}}^{3.6} \text { (in Pa s) at } 20^{\circ} \mathrm{C}
\end{aligned}
$$

The $\tau_{\mathrm{w}}$ and $\eta_{0}$ data of our PDMS prepolymers (square and diamond), the former being subjected to the MWD correction, are well described by Eqs. (3a) and (3b). This result suggests that the vinyl groups at the prepolymer ends (and a small degree of chemical modification for one prepolymer) do not significantly affect the relaxation behavior of the prepolymers.

\subsection{Relaxation Behavior of PDMS Gel}

For the as-prepared PDMS gel (containing the sol component), Fig. 3 shows the master curves of the $G^{\prime}$ and $G^{\prime \prime}$ data obtained from the dynamic oscillatory measurement (circles). The reference temperature is $T_{\mathrm{r}}=20^{\circ} \mathrm{C}$. The intensity

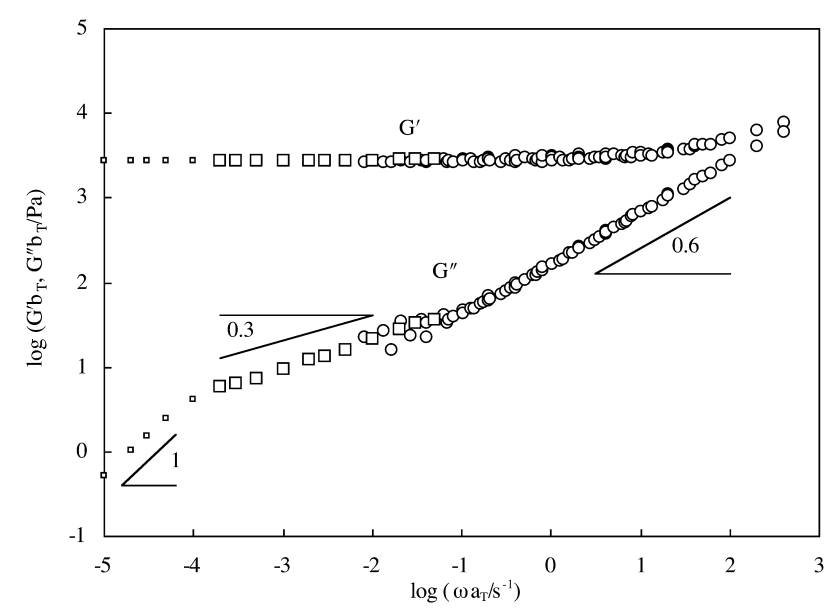

Fig. 3. Dependence of storage modulus $\left(G^{\prime}\right)$ and loss modulus $\left(G^{\prime \prime}\right)$ on the angular frequency $(\omega)$ measured for the as-prepared PDMS gel at $20{ }^{\circ} \mathrm{C}$. Circles indicate the data directly obtained from dynamic oscillatory tests, and squares show the data converted from the creep compliance data. reduction factor utilized in Fig. $3, b_{\mathrm{T}}=T_{\mathrm{r}} \rho_{\mathrm{r}} / T \rho$ with $\rho$ being the density and $T$ in the absolute temperature unit, was evaluated from the separately measured linear thermal expansion coefficient $\alpha_{\mathrm{L}}: \rho=\rho_{\mathrm{r}}\left\{1-\alpha_{\mathrm{L}}{ }^{3}\left(T-T_{\mathrm{r}}\right)\right\}$. In the range of $\omega$ examined in the dynamic measurements, $G^{\prime}$ decreases to the equilibrium plateau but $G^{\prime \prime}$ has not exhibited its terminal behavior characterized with the proportionality, $G^{\prime \prime} \propto \omega$. Thus, we conducted a creep test with an attempt of detecting the low- $\omega$ behavior missed in the dynamic measurement.

The creep compliance data measured at $t<10^{4} \mathrm{~s}$ are shown in Fig. 4. These data were converted to a retardation spectrum (through a fit shown with the solid curve) and further to the $G^{\prime}$ and $G^{\prime \prime}$ data with a method described in Appendix A. The resulting $G^{\prime}$ and $G^{\prime \prime}$ data are shown in Fig. 3 with squares. These data are in good agreement with those directly obtained from the dynamic measurements (circles) in the range of $\omega$ where the two sets of data are available.

In Fig. 3, our PDMS gel exhibits a plateau of $G^{\prime}$ in a wide range of $\omega<10^{0} \mathrm{~s}^{-1}$. The equilibrium modulus, $G_{\mathrm{e}}=2800 \mathrm{~Pa}$, is about two orders of magnitude smaller than that of usual "silicone rubbers", meaning that the double-liquid crosslinking reaction utilized in this study gave a very soft material. In addition, the PDMS gel exhibits a surprisingly small mechanical loss, $\tan \delta=G^{\prime \prime} / G^{\prime} \leq 10^{-2}$ at $\omega<10^{-1} \mathrm{~s}^{-1}$, which is hardly observed for usual rubbers (except for a few cases ${ }^{7)}$ ). These features are discussed later in relation to gel network structure.

Now, we focus on the $\omega$ dependence of $G^{\prime \prime}$ that characterizes the relaxation mode distribution of the PDMS gel. (This distribution is less clearly resolved for $G^{\prime}$ because the equilibrium plateau of $G^{\prime}$ masks changes of $G^{\prime}$ due to those relaxation modes.) $G^{\prime \prime}$ exhibits a characteristic power-law decrease with decreasing $\omega$ to $10^{-1} \mathrm{~s}^{-1}$,

$$
G^{\prime \prime} \propto \omega^{n} \text { with } n \cong 0.6 \text { at } \omega=10^{3}-10^{-1} \mathrm{~s}^{-1}
$$

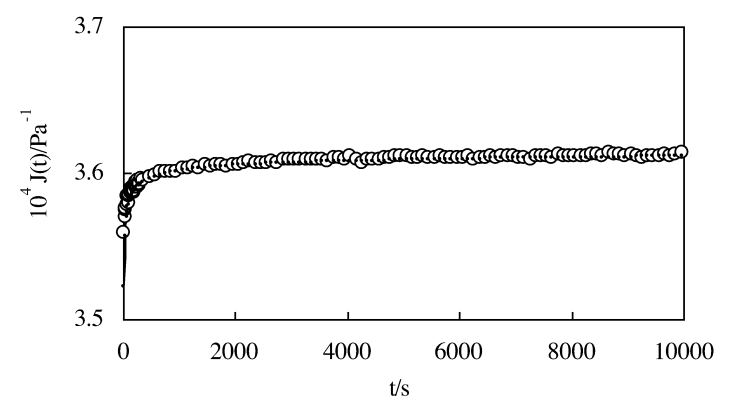

Fig. 4. Creep compliance of the as-prepared PDMS gel at $20^{\circ} \mathrm{C}$. Solid curve indicates the fit with a set of four retardation times and intensities. For details, see Appendix A. 
On a further decrease of $\omega, G^{\prime \prime}$ becomes less dependent on $\omega$ and this behavior can be approximately described by the other form of power-law,

$$
G^{\prime \prime} \propto \omega^{n^{\prime}} \text { with } n^{\prime} \cong 0.3 \text { at } \omega=10^{-1}-10^{-4} \mathrm{~s}^{-1}
$$

Thus, the PDMS gel exhibits fast and slow relaxation processes that govern the behavior of $G^{\prime \prime}$ at respective ranges of $\omega$.

Here, a comment needs to be made for the $G^{\prime \prime}$ data converted from the $J(t)$ data (squares in Fig. 3). The $G^{\prime \prime}$ data in a range of $\omega>10^{-4} \mathrm{~s}^{-1}$ (large squares) were accurately obtained and their power-law behavior (Eq. (5)) was detected with little uncertainties, because the $J(t)$ data were measured in the corresponding range of $t\left(<10^{4} \mathrm{~s}\right)$. However, as explained in Appendix A, the real $\omega$ dependence of $G^{\prime \prime}$ at $\omega<10^{-4} \mathrm{~s}^{-1}$ (small squares) may be somewhat weaker than that shown in Fig. 3 because the $J(t)$ data at $t>10^{4} \mathrm{~s}$ determining this dependence were not obtained in our creep test. This point is considered in the estimation of the terminal relaxation time described below.

In Fig. 3, $G^{\prime \prime}$ decreases in proportion to $\omega$ and the terminal behavior of a viscoelastic solid (gel) prevails at very low $\omega$ $\left(<10^{-4} \mathrm{~s}^{-1}\right)$. From these $G^{\prime \prime}$ data, the terminal relaxation time of the slow process is estimated to be $\cong 5000 \mathrm{~s}$. However, at $\omega<10^{-4} \mathrm{~s}^{-1}$, the real $\omega$ dependence of $G^{\prime \prime}$ may be somewhat weaker than that shown in Fig. 3, as mentioned above. Thus, this estimate is to be regarded as the minimum and the real terminal relaxation time $\tau_{\mathrm{w}}$ is specified as

$$
\tau_{\mathrm{w}} \geq 5000 \mathrm{~s}
$$

Finally, we should note that $G^{\prime}$ decreases to its equilibrium (terminal) plateau at $\omega \cong 10^{0} \mathrm{~s}^{-1}$ while the terminal relaxation behavior of $G^{\prime \prime}$, if any, is observed at much lower $\omega\left(<10^{-4} \mathrm{~s}\right)$. This superficial inconsistency of the $\omega$ ranges for respective terminal behavior is related to an intensity of the slow relaxation process: This process, detected for $G^{\prime \prime}$, has the intensity negligibly smaller than the equilibrium plateau of $G^{\prime}$ $\left(G_{\mathrm{e}}=2800 \mathrm{~Pa}\right)$, thereby allowing $G^{\prime}$ to practically approach its equilibrium plateau at $\omega \cong 10^{0} \mathrm{~s}^{-1}$ where the terminal relaxation has not been attained. Accordingly, $G^{\prime \prime}$ (not contributed from this equilibrium elasticity) is orders of magnitudes smaller than $G^{\prime}$ at low $\omega$; see Fig. 3 .

\subsection{Characterization of Gel Network Structure}

For characterization of our PDMS gel network, we conducted the swelling experiment in toluene with the method explained earlier. The sol component was fully extracted from the gel, and the sol fraction in the as-prepared gel (before the extraction) was determined to be

$$
f_{\text {sol }}=0.58
$$

Figure 5 shows the GPC chromatogram obtained for the sol component (solid curve) and the neat PDMS prepolymer (with no chemical modification; dotted curve). The average molecular weight of the sol component was

$$
M_{\mathrm{n}}=11.9 \times 10^{3} \text { and } M_{\mathrm{w}}=50.3 \times 10^{3}
$$

This $M_{\mathrm{w}}$ value is larger than $M_{\mathrm{w} \text {,pre }}$ of the prepolymer only by a factor $\cong 2$, indicating that a large amount of the prepolymer was left unreacted in the as-prepared gel and the reaction efficiency was considerably low. The sol also has a high- $M$ tail almost reaching $M=10^{6}$ but such high- $M$ component is very minor in the sol; $c f$. Fig. 5.

For the gel after the full extraction of the sol, the volumes $V$ and $V_{0}$ were determined as a mass/density ratio in the swollen equilibrium state and the fully dried state, respectively. From the equilibrium swelling ratio defined by $Q_{\text {eq }}=V / V_{0}(=21.0)$, we may estimate the molecular weight between the crosslinks $M_{\mathrm{c}}$ on the basis of the Flory-Rehner theory. ${ }^{16,26)}$ Since our gel was crosslinked in the presence of a sol (unreacted diluent), the dry gel network after the full extraction of the sol is collapsed compared to its natural state by a factor of $f_{\text {gel }}=1-f_{\text {sol }}$ $=0.42$ (gel fraction in the as-prepared gel). Correspondingly, for this dry gel, a change in the elastic free energy on swelling to a magnitude $Q$ can be expressed as $\Delta F_{\mathrm{el}}=\left(R T v_{\mathrm{e}} / 2\right)\left[3\left(Q f_{\mathrm{gel}}\right)^{2 / 3}\right.$ $\left.-3-\ln \left(Q f_{\text {gel }}\right)\right]$ with $R$ and $v_{\mathrm{e}}$ being the gas constant and the number density of active gel strands (in mole unit), respectively. ${ }^{16,26)}$ Utilizing this expression in the Flory-Rehner theory (that originally considered the crosslinking reaction leaving no sol and giving $f_{\text {gel }}=1$ ), we obtain

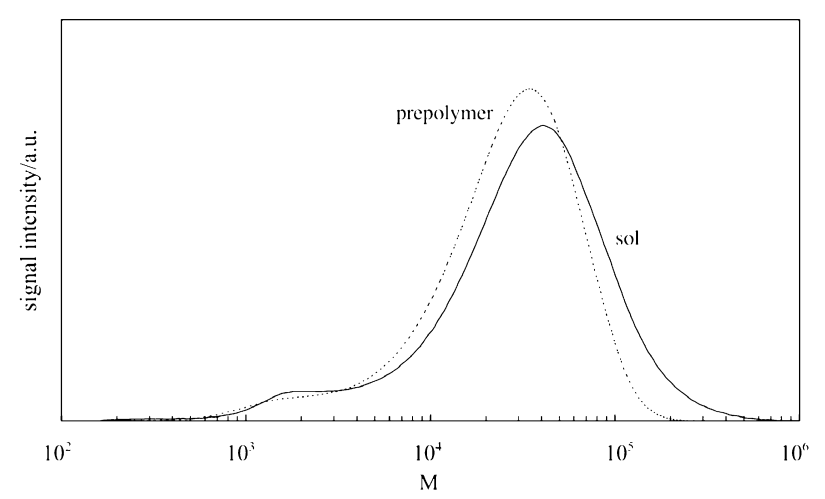

Fig. 5. Molecular weight distributions of the PDMS prepolymer (dotted curve) and sol fraction in the as-prepared PDMS gel (solid curve). 


$$
M_{\mathrm{c}}=\frac{v_{\mathrm{s}}}{v_{\mathrm{gel}}} Q_{\mathrm{eq}}^{5 / 3} f_{\mathrm{gel}}^{2 / 3}\left(\frac{1}{2}-\chi_{\mathrm{PDMS} / \mathrm{Tol}}\right)^{-1} \cong 260 \times 10^{3}
$$

Here, $v_{\mathrm{s}}$ and $v_{\text {gel }}$ represent the molar volume of toluene (solvent) and the specific volume of the PDMS gel, respectively, and $\chi_{\mathrm{PDMS} / \mathrm{Tol}}$ is the interaction parameter between PDMS and toluene. The $\chi_{\text {PDMS/Tol }}$ value $(=0.463)$ was evaluated by extrapolating the concentration dependent $\chi_{\mathrm{PDMS} / \mathrm{Tol}}$ data in literature $^{27)}$ to the PDMS concentration in our swollen gel.

We also utilized the conventional expression of plateau modulus $^{16,25)} G_{\mathrm{e}}$ to estimate $M_{\mathrm{c}}$ as

$$
M_{\mathrm{c}}=\frac{\rho f_{\mathrm{gel}} R T}{G_{\mathrm{e}}} \cong 340 \times 10^{3} \quad\left(G_{\mathrm{e}}=2800 \mathrm{~Pa}\right)
$$

(The factor $\rho f_{\text {gel }}$ represents the concentration of the network strands in the as-prepared gel; the sol chains behave as a diluent for these strands at $\omega \rightarrow 0$.) This $M_{\mathrm{c}}$ value is satisfactorily close to that obtained from the swelling experiment (Eq. (9)).

The above evaluation of $M_{\mathrm{c}}$ includes some uncertainty because it does not account for the effects of the crosslinking point fluctuation ${ }^{16,17)}$, trapped entanglements ${ }^{18,19)}$, and dangling chains. However, the $M_{c}$ values given in Eqs. (9) and (10) are an order of magnitude larger than the molecular weight of the PDMS prepolymers $\left(M_{\mathrm{w}, \mathrm{pre}} \cong 35 \times 10^{3}\right)$, allowing us to conclude that the effective strand in our PDMS gel is composed of $\sim$ ten prepolymer chains on average.

Since the upper route of reaction shown in Scheme 1 (grafting reaction) occurs more frequently than the lower route $^{22)}$ and the efficiency of reaction is considerably low (as noted from the large $f_{\text {sol }}$ value; Eq. (7)), the gel strand would be a scarce comb-like strand that is composed of linearly linked prepolymers and a few dangling chains grafted thereto. (The number of prepolymers per strand, ten, includes those dangling chains). Because of the low efficiency of the reaction, the majority of the dangling chains would be the prepolymers that reacted only at one end. These dangling prepolymers could have an important effect on the viscoelastic properties of the gel, as discussed below.

The crosslinking reaction in bulk phase usually leads to significant formation of the trapped entanglements, and these entanglements effectively decrease $M_{\mathrm{c}}$ (thereby giving $M_{\mathrm{c}}<$ $M_{\text {pre }}$ ) and enhance the mechanical loss at low $\omega$. Our PDMS gel was crosslinked in the bulk phase but still has $M_{\mathrm{c}} \gg M_{\text {pre }}$ and a surprisingly small mechanical loss, $\tan \delta=G^{\prime \prime} / G^{\prime} \leq 10^{-2}$ at $\omega<10^{-1} \mathrm{~s}^{-1}$. These unexpected features can be related to the fairy short sol chains and dangling prepolymers existing in the as-prepared gel. The sol fraction during the reaction should have been larger than that after the reaction $\left(f_{\text {sol }}=0.58\right.$; Eq. (7)), and such concentrated sol chains could have significantly diluted the trapped entanglements during the reaction, as similar to the situation for the solution-crosslinked rubbers. ${ }^{11)}$ The steric repulsion between the dangling prepolymers would further suppress the formation of the trapped entanglements. In addition, the fairly short sol chains and dangling prepolymers (having $M_{\mathrm{w}, \mathrm{sol}} \cong 2 M_{\eta}$ and $M_{\mathrm{w} \text {,pre }} \cong 1.4 M_{\eta}$ ) would have relaxed quickly and hardly enhanced the mechanical loss at low $\omega$. The unexpectedly large $M_{\mathrm{c}}$ value and small tan $\delta$ value of our PDMS gel can be attributed to these effects of the short sol chains and dangling prepolymers.

\subsection{Relaxation Mechanisms in PDMS Gel}

The as-prepared PDMS gel exhibits the fast and slow relaxation processes at $\omega>10^{-1} \mathrm{~s}^{-1}$ and $\omega<10^{-1} \mathrm{~s}^{-1}$, respectively; $c f$. Fig. 3 and Eqs. (4) and (5). In the followings, we consider several molecular mechanisms and examine if these mechanisms are responsible for the fast and slow processes.

\subsubsection{Role of sol chains and dangling chains in the fast process}

For the fast process occurring at $\omega>10^{-1} \mathrm{~s}^{-1}$, the mechanism to be considered first is the motion of the gel strands and sol/ dangling chains within the entanglement segment of the molecular weight $M_{\mathrm{e}}\left(=8.1 \times 10^{3}\right.$ for PDMS $\left.{ }^{24)}\right)$. This motion is free from any entanglement effect, and its characteristic time $\tau^{\circ}\left(M_{\mathrm{e}}\right)$ can be estimated in the following way.

As well known, the zero-shear viscosity and steady state compliance of monodisperse linear polymers scale with $M$ as ${ }^{24,25)}$

$$
\begin{aligned}
& \eta_{0} \propto M \text { for } M<M_{\eta}, \quad \eta_{0} \propto M^{3.5 \pm 0.2} \text { for } M>M_{\eta} \\
& J_{\mathrm{e}} \propto M \text { for } M<M_{\mathrm{J}}, \quad J_{\mathrm{e}} \propto M^{0} \text { for } M>M_{\mathrm{J}}
\end{aligned}
$$

Here, $M_{\eta}$ and $M_{\mathrm{J}}$ are the characteristic molecular weights for the onset of the entanglement effect on $\eta_{0}$ and $J_{\mathrm{e}}: M_{\eta}=$ $24.4 \times 10^{3}$ and $M_{\mathrm{J}}=61.0 \times 10^{3}$ for PDMS. ${ }^{24)}$ From Eqs. (11) and (12), the terminal relaxation time $\tau_{\mathrm{w}}=J_{\mathrm{e}} \eta_{0}$ scales with $M$ as

$$
\begin{aligned}
& \tau_{\mathrm{w}} \propto M^{2} \text { for } M<M_{\eta} \\
& \tau_{\mathrm{w}} \propto M^{4.5 \pm 0.2} \text { for } M_{\eta}<M<M_{\mathrm{J}} \\
& \tau_{\mathrm{w}} \propto M^{3.5 \pm 0.2} \text { for } M_{\mathrm{J}}<M
\end{aligned}
$$

The empirical Eq. (3a), obtained for $M>M_{\mathrm{J}}$, gives $\tau_{\mathrm{w}}\left(M_{\mathrm{J}}\right)=$ $1.0 \times 10^{-21} M_{\mathrm{J}}^{3.6} \cong 1.7 \times 10^{-4} \mathrm{~s}$. Combining this $\tau_{\mathrm{w}}\left(M_{\mathrm{J}}\right)$ value with 
Eq. (13b), we obtain $\tau_{\mathrm{w}}\left(M_{\eta}\right)=\left\{M_{\eta} / M_{\mathrm{J}}\right\}^{4.6} \tau_{\mathrm{w}}\left(M_{\mathrm{J}}\right) \cong 2.5 \times 10^{-6} \mathrm{~s}$. Applying Eq. (13a) to this $\tau_{\mathrm{w}}\left(M_{\eta}\right)$, we may estimate $\tau^{\circ}\left(M_{\mathrm{e}}\right)$ for the entanglement as

$$
\tau^{\mathrm{o}}\left(M_{\mathrm{e}}\right)=\left(\frac{M_{\mathrm{e}}}{M_{\eta}}\right)^{2} \tau_{\mathrm{w}}\left(M_{\eta}\right) \cong 2.8 \times 10^{-7} \mathrm{~s}
$$

Literature data ${ }^{28)}$ suggest that $G^{\prime}$ of well entangled homoPDMS chains at $20^{\circ} \mathrm{C}$ levels off at the entanglement plateau at $\omega \sim 10^{6}-10^{7} \mathrm{~s}^{-1}$, i.e., at $t \sim 10^{-7}-10^{-6} \mathrm{~s}$, lending support to this estimate.

For our PDMS gel, the fast relaxation process ends at $\omega \sim$ $10^{-1} \mathrm{~s}^{-1}<<1 / \tau^{\circ}\left(M_{\mathrm{e}}\right) ; c f$. Fig. 3 . Thus, this process should involve motion of a portion of PDMS chains much larger than the entanglement segment, although the motion within the entanglement segment should have certainly occurred at high $\omega\left(>10^{6} \mathrm{~s}^{-1}\right)$ not covered in our experiment.

Similarly, we can estimate the characteristic time $\tau_{\text {sol }}$ for the global motion of the PDMS sol chains. The majority of the sol chains has $M_{\mathrm{w}, \mathrm{sol}}=50.3 \times 10^{3}$ (Eq. (8)), which is between $M_{\eta}$ and $M_{\mathrm{J}}$. Thus, we can follow the above method to estimate $\tau_{\text {sol }}$ as

$$
\tau_{\text {sol }} \cong 3 \times\left(\frac{M_{\mathrm{w}, \mathrm{sol}}}{M_{\mathrm{J}}}\right)^{4.6} \tau_{\mathrm{w}}\left(M_{\mathrm{J}}\right) \cong 2.1 \times 10^{-4} \mathrm{~s}
$$

Here, the front factor of 3 accounts for the retardation of the motion of entangled linear chains in a matrix of much longer chains and/or gel network. ${ }^{29)}$ The corresponding frequency, $1 / \tau_{\text {sol }}$ $\cong 5 \times 10^{3} \mathrm{~s}^{-1}$, is too large compared to the terminal frequency of the fast relaxation process, $\omega \cong 10^{-1} \mathrm{~s}^{-1}$. The sol has the high- $M$ tail up to $10^{6}$ (cf. Fig. 5), and $1 / \tau_{\text {sol }} \cong 10^{-1} \mathrm{~s}^{-1}$ for linear sol chains of $M=10^{6}$. Scheme 1 suggests that the crosslinking reaction could have also produced star-branched high- $M$ sol chains (or a fragment of network), and these star chains would have even smaller $1 / \tau_{\text {sol }}$. However, such high- $M$ chains are very minor in the sol ( $c f$. Fig. 5) and could have just a secondary contribution, at best, to the fast process characterized with the power-law behavior, Eq. (4). Thus, the dominant part of this process cannot be attributed to the motion of the sol chains, although the majority of these chains should have significantly contributed to $G^{\prime \prime}$ at $\omega \geq 1 / \tau_{\text {sol }}$ (majority) $\cong 5 \times 10^{3} \mathrm{~s}^{-1}$.

We also need to examine a contribution from dangling chains that would have formed mainly through the upper reaction route shown in Scheme 1. For high- $M$ dangling chains (with $M>>M_{\eta}$ ), the relaxation mode distribution should be close to that of highly entangled star-branched chains. Such star chains exhibit a broad terminal relaxation mode distribution, and their $G^{\prime \prime}$ data reported in literature ${ }^{30,31)}$ can be approximately cast in a power low form,

$$
G_{\text {star }}{ }^{\prime \prime} \propto \omega^{n^{\prime \prime}} \text { with } n^{\prime \prime}=0.15-0.2 \text { for } \omega_{\mathrm{t}}<\omega<\omega_{\mathrm{R}}
$$

with $\omega_{\mathrm{t}}$ and $\omega_{\mathrm{R}}$ being the terminal relaxation frequency and Rouse frequency of the star arm, respectively. This power-law behavior is quite different from that seen for the fast relaxation process of the PDMS gel (Eq. (4)). In addition, Scheme 1 suggests that the molecular weight distribution is not significantly different for the sol chains and dangling chains. As judged from the GPC trace of the sol (Fig. 5), the high- $M$ (highly entangled) dangling chains would have been hardly formed in the gel. For these reasons, the fast process of the gel cannot be related to the high- $M$ dangling chains.

On the other hand, a considerable amount of the dangling prepolymers should have been formed in the gel, as explained earlier. Since the dangling prepolymers (with $M_{\text {pre }} \cong 1.4 M_{\eta}$ ) are just moderately entangled, their relaxation frequency would not be significantly different from that of the majority of the sol component, $\tau_{\text {sol }} \cong 2.1 \times 10^{-4} \mathrm{~s}$; Eq. (15). Thus, the dangling prepolymers hardly contribute to the dominant part of the fast process seen at $\omega=10^{3}-10^{-1} \mathrm{~s}^{-1}$, although they would have contributed to $G^{\prime \prime}$ at higher $\omega$, as similar to the situation for the majority of the sol chains.

\subsubsection{Role of gel strands in the fast process}

The gel strand would be a scarce comb-like strand having a few grafts and the majority of the grafts would be the dangling prepolymers, as discussed earlier. The dangling prepolymers should have fully relaxed at $\omega=10^{3}-10^{-1} \mathrm{~s}^{-1}$, as discussed above. Thus, the motion of the strand considered here is the motion of the backbone of the comb-like strand. Hereafter, this backbone is simply referred to as the strand.

Since the power-law behavior of $G^{\prime \prime}$ (Eq. (4)) is not identical but close to the behavior expected for a Rouse chain with fixed ends $^{29,32)}\left(G^{\prime \prime} \propto \omega^{1 / 2}\right)$, it is tempting to assign the fast process to the Rouse motion of the strand. However, for a gel network composed of monodisperse strands, we realize that the terminal behavior of $G^{\prime \prime}(\propto \omega)$ emerges immediately after $G^{\prime}$ reaches the equilibrium plateau on a decrease of $\omega$, as explained later in more details. Thus, we have to consider the gel network having the strand length distribution. For the Rouse network with this distribution, $G^{\prime}$ and $G^{\prime \prime}$ are described by $^{32)}$

$$
G^{\prime}(\omega)=\sum_{\mathrm{i}} \frac{C_{\mathrm{st}} w_{\mathrm{i}} R T}{M_{\mathrm{i}}}\left[1+\sum_{p \geq 1} \frac{\omega^{2}\left\{\tau_{\mathrm{R}}\left(M_{\mathrm{i}}\right)\right\}^{2} p^{-4}}{1+\omega^{2}\left\{\tau_{\mathrm{R}}\left(M_{\mathrm{i}}\right)\right\}^{2} p^{-4}}\right]
$$




$$
G^{\prime \prime}(\omega)=\sum_{\mathrm{i}} \frac{C_{\mathrm{st}} w_{\mathrm{i}} R T}{M_{\mathrm{i}}}\left[\sum_{p \geq 1} \frac{\omega \tau_{\mathrm{R}}\left(M_{\mathrm{i}}\right) p^{-2}}{1+\omega^{2}\left\{\tau_{\mathrm{R}}\left(M_{\mathrm{i}}\right)\right\}^{2} p^{-4}}\right]
$$

with

$$
\tau_{\mathrm{R}}\left(M_{\mathrm{i}}\right)=\tau^{*} M_{\mathrm{i}}^{2}
$$

Here, $C_{\mathrm{st}}$ is the total concentration of the gel strands, $w_{\mathrm{i}}$ is the weight fraction of the $i$-th strand having the molecular weight $M_{\mathrm{i}}$, and $\tau_{\mathrm{R}}\left(M_{\mathrm{i}}\right)$ is the longest Rouse relaxation time of the $i$-th strand. In Eq. (19), the front factor $\tau^{*}$ is regarded as an adjustable parameter representing a local time constant.

Assuming that the gel contains four components of strands with their $\left\{w_{\mathrm{i}}, M_{\mathrm{i}}\right\}$ distribution being summarized in Table I, we made a model calculation of $G^{\prime}$ and $G^{\prime \prime}$. In this calculation, $C_{\text {st }}$ was replaced by the actual concentration of the gel strand, $\rho f_{\text {gel }}\left(c f\right.$. Eq. (10)), and the $\left\{w_{\mathrm{i}}, M_{\mathrm{i}}\right\}$ distribution was chosen in a way that the calculated equilibrium modulus agreed with the data. As shown in Fig. 6 , the $G^{\prime}$ and $G^{\prime \prime}$ calculated for $\tau^{*}=$ $8.7 \times 10^{-14} \mathrm{~s}$ (solid curves) agree well with the data in the dominant part of the fast relaxation process. This result strongly suggests that the fast process is attributable to the Rouse motion of the gel strands having a considerably broad length distribution, although the actual distribution in the PDMS gel would not necessarily agree with that in the calculation. (The polydispersity index for the gel strands utilized in the calculation is $M_{\mathrm{w}} / M_{\mathrm{n}}=4.38$.)

To demonstrate the effect of polydispersity on the $G^{\prime}$ and $G^{\prime \prime}$ curves, we utilized the same $\tau^{*}$ value to conduct the calculation for the monodisperse strand with $M=M_{\mathrm{c}}=$ $340 \times 10^{3}$. The results are shown in Fig. 6 with the dotted curves. The $G^{\prime}$ calculated for the monodisperse strands is almost the same as that for the polydisperse strands and close to the data. However, for the monodisperse strands, $G^{\prime \prime}$ begins to decrease in proportion to $\omega$ as soon as $G^{\prime}$ reaches the

Table I. Parameters utilized in Rouse calculation shown in Fig. 6.

\begin{tabular}{cccc} 
i & $w_{\mathrm{i}}$ & $10^{-3} M_{\mathrm{i}}$ & $10^{2} \tau_{\mathrm{R}}\left(M_{\mathrm{i}}\right) / \mathrm{s}$ \\
\hline 1 & 0.14 & 85.0 & 0.0625 \\
2 & 0.35 & 340 & 1 \\
3 & 0.35 & 1360 & 16 \\
4 & 0.16 & 5440 & 256
\end{tabular}

equilibrium plateau and the power-law behavior seen at $\omega>$ $10^{-1} \mathrm{~s}^{-1}, G^{\prime \prime} \propto \omega^{\mathrm{n}}$ with $\mathrm{n} \cong 0.6$, cannot be reproduced. Thus, the polydispersity of the gel strands, naturally expected from Scheme 1, is essential for this power-law behavior to be observed in the plateau regime for $G^{\prime}$. (The high- $M$ component of the strands has a very small number fraction $\left(\propto w_{\mathrm{i}} / M_{\mathrm{i}}\right)$ and negligibly contributes to the equilibrium plateau of $G^{\prime}$. However, this component has a long relaxation time and thus significantly contribute to $G^{\prime \prime}$ at low $\omega$ where the lower- $M$ components have relaxed. This contribution of the high- $M$ component results in the power-law-like behavior seen for the solid curve in Fig. 6.)

Further details of the Rouse motion of the polydisperse strands can be examined for their relaxation time $\tau_{\mathrm{R}}$. The front factor $\tau^{*}$ utilized in the above calculation gives the Rouse time for the entanglement segment $\left(M_{\mathrm{e}}=8.1 \times 10^{3}\right)$ :

$$
\tau_{\mathrm{R}}\left(M_{\mathrm{e}}\right)=5.7 \times 10^{-6} \mathrm{~s}
$$

This $\tau_{\mathrm{R}}$ value is significantly longer than the intrinsic (entanglement-free) relaxation time of this segment, $\tau\left(M_{\mathrm{e}}\right)=$ $2.8 \times 10^{-7} \mathrm{~s}$ (Eq. (14)). Thus, the Rouse motion giving the power-law behavior in the fast relaxation process cannot be the intrinsic Rouse motion. Instead, the Rouse motion in the fast process is attributable to the constraint release $(\mathrm{CR})$ Rouse motion of the polydisperse gel strands, as discussed below.

The strands in our PDMS gel do not significantly form the trapped entanglement (permanent knots) among themselves, as discussed earlier. However, the large-scale motion of a gel strand (over a distance comparable to their dimension $R_{\mathrm{st}}$ )

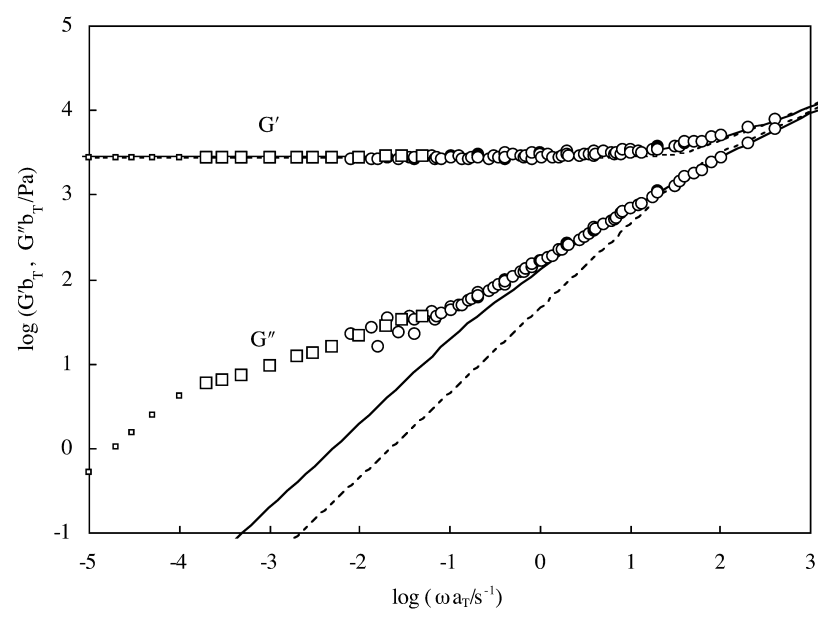

Fig. 6. Comparison of the Rouse calculation for polydisperse gel strands (solid curves) and the $G^{*}$ data (symbols). The dotted curves indicate the calculation for monodisperse strands. 
should be still constrained, in a short time scale, by the surrounding sol/dangling chains and other gel strands, both having $M>M_{\eta}$. In this sense, the strand is entangled with the sol/dangling chains and the other strands, as schematically shown in Fig. 7. Note that the strands can mutually entangle without forming a permanent knot, as illustrated in the bottomleft of Fig. 7.

Since the gel strand has no freedom of translational diffusion, the entanglement segment of this strand is allowed to achieve a local hopping motion and partially relax only when the entangling sol/dangling chains and/or other strands exhibit large scale motion. Accumulation of this local CR hopping results in the global relaxation over the distance close to the dimension of the gel strand, and this global CR relaxation has the Rouse-like character as established from extensive studies for blends of linear homopolymer chains. ${ }^{29)}$ Thus, the Rouse-like power-law behavior observed in the fast relaxation process is attributable to the global CR relaxation of individual strands (having a considerable polydispersity), although the sol chains and dangling prepolymers may have secondary contributions to this process.

Within this molecular scenario, the Rouse time $\tau_{\mathrm{R}}\left(M_{\mathrm{e}}\right)$
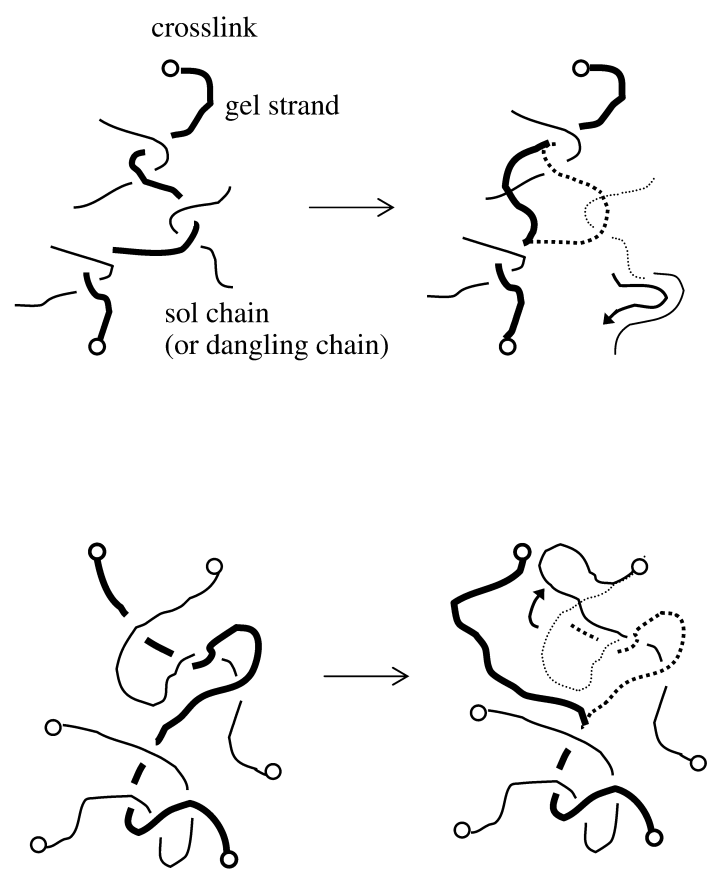

Fig. 7. Schematic illustration of constraint release process for a gel strand. In the top panel, motion of the sol chain or dangling chain (thin curve) activates the local constraint release (CR) hopping of a focused gel strand (backbone of the scarce comb-like strand; thick curve). In the bottom panel, the local CR hopping of the focused strand (thick curve) is activated by motion of a surrounding gel strand (thin curve) that is entangled, without forming a permanent knot, with the focused strand. obtained for the entanglement segment (Eq. (20)) is regarded to be the time required for the local CR-hopping of this segment. In the as-prepared PDMS gel, this time should be determined by the sol chains as well as the gel strands/ dangling prepolymers. A contribution of the sol chains to this local CR time can be viscoelastically tested for the dry gel after full extraction of the sol chains. This test is an interesting subject of future work.

\subsubsection{Slow process}

The slow relaxation process of the as-prepared PDMS gel is characterized with the power-law behavior (Eq. (5)) and the terminal relaxation frequency $\omega_{\mathrm{r}}=1 / \tau_{\mathrm{w}} \leq 2 \times 10^{-4} \mathrm{~s}^{-1}$ (Eq. (6)). If linear sol chains are to have this $\omega_{\mathrm{r}}$, their molecular weight should be of the order of $10^{7}$ or more, as judged from Eq. (3). No such high- $M$ chains are seen in the GPC trace (Fig. 5), allowing us to conclude that the slow relaxation is not attributed to the sol chains. Similarly, $\omega_{\mathrm{r}}$ appears to be too small for dangling chains/star-like sol chains of reasonable molecular weights. In addition, the power-law behavior observed for the slow process (Eq. (5)) is somewhat different from that expected for these chains (Eq. (16)). Thus, we may conclude that the dangling chains/star-like sol chains have just a secondary contribution, at best, to the slow relaxation process of the PDMS gel.

From this argument, the slow process should be mainly related to the gel strands themselves (backbone of the scarce comb-like strand having a few grafts). Since this process is orders of magnitude slower than the fast process assigned as the Rouse-CR relaxation of individual gel strands, we may attribute the slow process to thermal fluctuation of the crosslinking points $(\mathrm{CP})$ that is equivalent to cooperative $\mathrm{CR}$ motion of many gel strands connected at $\mathrm{CP}$ : The viscoelastic mode distribution associated to this fluctuation changes with the distribution of the strand length (as analogous to the normal-mode vibration of a jungle-gym-like cage) but is surely different from the Rouse mode distribution (giving $\left.G^{\prime \prime} \propto \omega^{1 / 2}\right)$ characteristic to an end-fixed linear strand. Unfortunately, at this moment, we cannot calculate the mode distribution for the CP fluctuation mechanism because of the lack of the detailed knowledge about the strand length distribution. It is interesting to calculate this mode distribution for the gel network of a known strand length distribution (such as the network prepared through the end-crosslinking reaction in fairly dilute solutions) and compare the result with the observed mode distribution of the slow process. These calculation/comparison are considered as an important subject of future work. 


\section{CONCLUDING REMARKS}

We have examined linear viscoelastic behavior of a PDMS gel prepared with a double-liquid crosslinking reaction of vinyl-terminated linear PDMS prepolymers in bulk. The asprepared gel, containing the sol chains of a fraction $f_{\text {sol }}=0.58$, behaved as a very soft viscoelastic solid. Analysis of the equilibrium modulus and swelling ratio suggested that the gel strand had $M_{\mathrm{c}} \cong 340 \times 10^{3}$ and corresponded to $\sim$ ten prepolymers. Thus, the double-liquid crosslinking reaction gave a very scarce gel structure that hardly contained trapped entanglements, possibly because the sol chains/dangling prepolymers effectively diluted the trapped entanglements during the reaction.

This scarce PDMS gel exhibited fast and slow relaxation processes. The fast process was associated with the power-law behavior, $G^{\prime \prime} \propto \omega^{\mathrm{n}}$ with $\mathrm{n} \cong 0.6$. This process is attributable to the Rouse-like constraint release (CR) motion of polydisperse gel strands being activated by the motion of the surrounding sol chains/dangling chains/ gel strands. (The polydispersity is essential for the gel to exhibit this power-law behavior of $G^{\prime \prime}$ in the plateau regime of $G^{\prime}$.) The slow process was associated with the other type of power-law behavior, $G^{\prime \prime} \propto \omega^{\mathrm{n}^{\prime}}$ with $\mathrm{n}^{\prime} \cong$ 0.3 . This process may be related to thermal fluctuation of the crosslinking points that is equivalent to cooperative $\mathrm{CR}$ motion of many gel strands connected at these points.

\section{Acknowledgements}

We acknowledge with thanks the invaluable supports from Messrs. H. Ichikawa, T. Hirano, N. Higeta and H. Kawagoe of Toray Research Center Inc.

\section{Appendix A: Evaluation of $G^{\prime}$ and $G$ " from $J(t)$ data}

For a viscoelastic solid, the creep compliance $J(t)$ is expressed in terms of the equilibrium compliance $J_{\mathrm{e}}^{\infty}\left(=1 / G_{\mathrm{e}}\right)$ and the retardation spectrum $\left\{J_{\mathrm{p}}, \lambda_{\mathrm{p}}\right\}$ as

$$
J(t)=J_{\mathrm{e}}^{\infty}-\sum_{\mathrm{p}=1}^{N} J_{\mathrm{p}} \exp \left(-\frac{t}{\lambda_{\mathrm{p}}}\right)
$$

(Here, a discrete spectrum composed of $N$ retardation modes is assumed.) Thus, we can evaluate the retardation times $\lambda_{\mathrm{p}}$ and retardation intensities $J_{\mathrm{p}}(p=1-N)$ by fitting the $J(t)$ data with Eq. (A1).

In the actual fitting procedure for the $J(t)$ data of the PDMS gel (Fig. 4), we first determined $J_{\mathrm{e}}^{\infty}\left(=[J(t)]_{\tau \rightarrow \infty}\right)$ : Since the $J(t)$ data were constant within experimental scatter at $t>8 \times 10^{3} \mathrm{~s}$ (cf. Fig. 4), the $J(t)$ value at those $t$ was utilized as the best estimate of $J_{\mathrm{e}}^{\infty}\left(=3.61 \times 10^{-4} \mathrm{~Pa}^{-1}\right)$. Then, a difference, $J_{\mathrm{e}}^{\infty}-J(t)$, was fitted with a sum of exponential decay terms, $J_{\mathrm{p}} \exp \left(-t / \lambda_{\mathrm{p}}\right)$ with $p=1-N$. (This fitting was made from long $t$ to short $t$ with Tobolsky's Procedure X method. ${ }^{25)}$ ) The fit for $N=4$ excellently reproduced the $J(t)$ data, as shown in Fig. 4 with the solid curve. The longest retardation time in this fitting was $\lambda_{1}=5.4 \times 10^{3} \mathrm{~s}$. Equally good fit was achieved for $N=6$ and 8 , suggesting that the fitting for $N=4$ was made with satisfactory accuracy.

From the $J_{\mathrm{e}}^{\infty}$ value and the retardation spectrum $\left\{J_{\mathrm{p}}, \lambda_{\mathrm{p}}\right\}$ for $N=4$, the real and imaginary components of the complex compliance, $J^{\prime}(\omega)$ and $J^{\prime \prime}(\omega)$, are straightforwardly obtained as

$$
\begin{aligned}
& J^{\prime}(\omega)=J_{\mathrm{g}}+\sum_{\mathrm{p}=1}^{4} J_{\mathrm{p}} \frac{1}{1+\left(\omega \lambda_{\mathrm{p}}\right)^{2}} \text { with } J_{\mathrm{g}}=J_{\mathrm{e}}^{\infty}-\sum_{\mathrm{p}=1}^{4} J_{\mathrm{p}} \\
& J^{\prime \prime}(\omega)=\sum_{\mathrm{p}=1}^{4} J_{\mathrm{p}} \frac{\omega \lambda_{\mathrm{p}}}{1+\left(\omega \lambda_{\mathrm{p}}\right)^{2}}
\end{aligned}
$$

These components are trivially converted to $G^{\prime}(\omega)$ and $G^{\prime \prime}(\omega)$ as

$$
G^{\prime}(\omega)=\frac{J^{\prime}(\omega)}{J^{\prime}(\omega)^{2}+J^{\prime \prime}(\omega)^{2}}, \quad G^{\prime \prime}(\omega)=\frac{J^{\prime \prime}(\omega)}{J^{\prime}(\omega)^{2}+J^{\prime \prime}(\omega)^{2}}
$$

These $G^{\prime}(\omega)$ and $G^{\prime \prime}(\omega)$ data are shown in Fig. 3 with the squares.

Here a comment needs to be made for changes of $J(t)$ that might occur in a range of $t>10^{4} \mathrm{~s}$ not covered in our creep test. Although the above fitting (with $\lambda_{1}=5400 \mathrm{~s}$ ) satisfactorily described the data at $t \leq 10^{4} \mathrm{~s}$ and thus the $G^{\prime}(\omega)$ and $G^{\prime \prime}(\omega)$ are accurately obtained in the corresponding range of $\omega>10^{-4} \mathrm{~s}$, the $\omega$ dependence of $G^{\prime \prime}(\omega)$ at $\omega<10^{-4} \mathrm{~s}^{-1}$ might be weaker than that shown in Fig. 3 (small squares) if the PDMS gel had undetectably slow retardation modes (with $\lambda_{1}>5400 \mathrm{~s}$ ) and its $J(t)$ increases with $t>10^{4} \mathrm{~s}$. For this case, the terminal relaxation time is longer than the time, $\cong 5000 \mathrm{~s}$, obtained from the data in Fig. 3. In other words, $5000 \mathrm{~s}$ is to be interpreted as the minimum for the actual terminal relaxation time. (From the $G^{\prime}(\omega)$ and $G^{\prime \prime}(\omega)$ data reliably obtained at $\omega>10^{-4} \mathrm{~s}^{-1}$, we can unequivocally conclude that the terminal relaxation time is not shorter than $5000 \mathrm{~s}$.) 


\section{Appendix B: Correction of molecular weight distribution for the relaxation time}

For entangled linear polymers with a unimodal molecular weight distribution, the zero-shear viscosity $\eta_{0}$ is essentially determined by the weight-average molecular weight $M_{\mathrm{w}}$ and hardly depends on the molecular weight distribution (MWD) while the steady state compliance $J_{\mathrm{e}}$ is quite sensitive to MWD. ${ }^{25)}$ Thus, the terminal relaxation time $\tau_{\mathrm{w}}=J_{\mathrm{e}} \eta_{0}$ is also sensitive to MWD. This should be the case also for our PDMS prepolymers.

For a correction of this MWD effect, we utilized an empirical equation reported by Agarwal, ${ }^{33)}$

$$
J_{\mathrm{e}} \propto \frac{M_{\mathrm{z}} M_{\mathrm{z}+1}}{M_{\mathrm{w}} M_{\mathrm{n}}}=\left(\frac{M_{\mathrm{z}+1}}{M_{\mathrm{z}}}\right)\left(\frac{M_{\mathrm{z}}}{M_{\mathrm{w}}}\right)^{2}\left(\frac{M_{\mathrm{w}}}{M_{\mathrm{n}}}\right)
$$

Here, $M_{\mathrm{z}+1}, M_{\mathrm{z}}$, and $M_{\mathrm{n}}$ are the $\mathrm{z}+1, \mathrm{z}$, and number-averaged molecular weights, respectively. Linear polymers of unimodal MWD often have $M_{\mathrm{z}+1} / M_{\mathrm{z}} \cong M_{\mathrm{z}} / M_{\mathrm{w}} \cong M_{\mathrm{w}} / M_{\mathrm{n}}$. For such polymers, Eq. (B1) is reduced to $J_{\mathrm{e}} \propto\left(M_{\mathrm{w}} / M_{\mathrm{n}}\right)^{4}$. Thus, we multiplied a factor $\left(M_{\mathrm{w}} / M_{\mathrm{n}}\right)^{-4}$ to the measured $\tau_{\mathrm{w}}$ of the PDMS prepolymers to make the correction for the MWD effect. These corrected $\tau_{\mathrm{w}}$ data are shown in Fig. 2a with the square and diamond symbols. (The empirical equation by Mills, ${ }^{34)}$ $J_{\mathrm{e}} \propto\left(M_{\mathrm{z}} / M_{\mathrm{w}}\right)^{3,7}$, gave nearly the same magnitude of correction.)

\section{REFERENCES}

1) Chambon F, Winter HH, J Rheol, 31, 683 (1987).

2) Tixier T, Tordjeman PH, Cohen-Solal G, Mutin PH, J Rheol, 48, 39 (2004).

3) Adolf D, Martin JE, Macromolecules, 24, 6721 (1991).

4) Oikawa H, Murakami K, Macromolecules, 24, 1117 (1991).

5) Horkay F, Hecht AM, Zrinyi M, Geisslar E, Polymer Gels and Networks, 4, 451 (1996).

6) Schimmel KH, Heinrich G, Colloid Polym Sci, 269, 1003 (1991).

7) Patel SK, Malone S, Cohen C, Gillmor JR, Colby RH, Macromolecules, 25, 5241 (1992).

8) Mark JE, Acc Chem Res, 27, 271 (1994).
9) Larsen AL, Hansen K, Sommer-Larsen P, Hassager O, Bach A, Ndoni S, J $\phi$ rgensen M, Macromolecules, 36, 10063 (2003).

10) Soni VK, Stein RS, Macromolecules, 23, 5257 (1990).

11) Urayama K, Kohjiya S, Eur Phys J B, 2,75 (1998).

12) Urayama K, Kawamura T, Hirata Y, Kohjiya S, Polymer, 39, 3827 (1998).

13) Urayama K, Kawamura T, Kohjiya S, Macromolecules, 34, 8252 (2001).

14) Urayama K, Kawamura T, Kohjiya S, Macromolecules, 34, 8261 (2001).

15) Urayama K, Miki T, Takigawa T, Kohjiya S, Chem Mater, 16, 173 (2004).

16) Flory PJ, "Principles of Polymer Chemistry", Cornell Univ Press, Ithaca, New York, 1953.

17) James HM, Guth EJ, J Chem Phys, 15, 669 (1947).

18) Langley NR, Macromolecules, 1, 348 (1968).

19) Dossin LM, Graessley WW, Macromolecules, 12, 123 (1979).

20) Murakami M, Oikawa H, Suh KD, Nihon Reoroji Gakkaishi (J Soc Rheol, Jpn), 17, 13 (1989).

21) Edwards SF, Vilgis TA, Polymer, 47, 483 (1986).

22) Macosko CW, Benjamin GS, Pure Appl Chem, 53, 1505 (1981).

23) Ressia JA, Villar MA, Vallés EM, Polymer, 41, 6885 (2000).

24) Graessley WW, Adv Polym Sci, 16, 1 (1974).

25) Ferry JD, "Viscoelastic Properties of Polymers", 3rd Ed, Wiley, New York, 1980.

26) Flory PJ, Rehner J, J Chem Phys, 11, 521 (1943).

27) Schuld N, Wolf BA, "Polymer Handbook", 4th Ed., edited by Brandrup J, Immergut EH, Grulke EA, Wiley, New York, Volume2,1999, chapter VII, p253

28) Rahalkar RR, Lamb J, Harrison G, Barlow AJ, Faraday Symp Chem Soc, 18, 103 (1983).

29) Watanabe H, Progr Polym Sci, 24, 1253 (1999).

30) Fetters LJ, Kiss AD, Pearson DS, Quack GF, Vitus FJ, Macromolecules, 26, 647 (1993).

31) Watanabe H, Matsumiya $Y$, Inoue $T$, Macromolecules, 35, 2339 (2002).

32) Doi M and Edwards SF, "The Theory of Polymer Dynamics", Clarendon, Oxford, 1986.

33) Agarwal PK, Macromolecules, 12, 342 (1979).

34) Mills NJ, Nature (London), 219, 1249 (1968). 